BLAKE's later biochemorphic reports are to be found mainly in The Journal of Anatomy and Physiology, The Journal of Physiology, and in Comptes rendus hebdomadaires des séances de l'Académie des sciences.

An accuunt of James Blake may be found in Henry Harri's California's Medical Story (San Francisco, J.W. Stacey, 1932, p. 342-7). A review of his clinical career appears in California and Western Medicine (47, 405-7, 1937).

\title{
Das «Buch über die Wiederkäuer» («Merycologia») von Johann Conrad Peyer, eine der geschichtlichen Grundlagen der heutigen Haustierphysiologie
}

\author{
Von Nikolaus Mani, Basel
}

Ein beträchtlicher Teil der biologischen Forschung des 17. Jahrhunderts betrifft die Anatomie und Physiologie des Magendarmkanals. Aselli entdeckte 1622 die Chylusgefäße und begründete in zahlreichen Versuchen die experimentelle Methode für das Studium der Darmresorption. Pecquet beschrieb 1647 den Ductus thoracicus und die Cisterna chyli beim Hunde. Mit dieser Entdeckung versetzte er der noch immer fest verwurzelten GaLevischen Resorptionstheorie den Todesstoß. Nicht den Gekrösevenen, sondern ausschließlich den Venae lacteae, den Chylusgefäßen, schrieb man nun die Aufnahme des Nahrungsbreies zu.

Die Entdeckung des Blutkreislaufes durch Harvey (1628) zeigte die Blutversorgung aller Organe in völlig neuem Lichte und nötigte die Ärzte und Naturwissenschafter zu einer generellen Überprüfung der GALENischen Physiologie.

Die anatomische Beschreibung der Ausführungsgänge des Pankreas (Wirsung 1641) und der Speicheldrüsen (Wharton 1655, Stensen 1669) lenkte das Interesse der Forscher auf das Studium der Drüsenfunktion und Drüsensekrete.

Dann folgte das klassische Werk Malpighis über die Struktur der Eingeweide. Die altehrwürdigen, von Aristoteles, Erasistratos und Galen übereinstimmend zu den blutartigen Körperteilen gezählten Viscera wurden mit einem Schlage in die neuentdeckte Gruppe der Drüsenorgane eingereiht. 
In diese Zeit des Umbruches vom Alten zum Neuen, die gekennzeichnet ist durch intensivste anatomische und physiologische Forschung, fällt das Wirken der Schaffhauser Ärzteschule. Ihr Begründer und zugleich bedeutendster Vertreter war Johann JaKob Wepfer (1620-1695), Stadtarzt in Schaffhausen. Ihm zur Seite arbeiteten in erfolgreicher Forschertätigkeit seine beiden hervorragenden Schüler Johann Conrad Brunner (1653-1727) und Johann Conrad Peyer (1653-1712). Die Vertreter der Schaff hauser medizinischen Schule haben in der zweiten Hälfte des 17. Jahrhunderts einen bedeutenden Beitrag zur Erforschung des Magendarmtraktes geleistet.

Neben toxikologischen ${ }^{1}$ und gehirnanatomischen Untersuchungen erkannte WePFER im Jahre 1664, also zwei Jahre vor MALPIGHI ${ }^{2}$, den lappigen Aufbau der Schweineleber. Er erwog die Frage, ob die Leber nun ebenfalls zu den Drüsen zu rechnen sei.

WEPFER hat auch zuerst die Glandulae intestinales und duodenales erkannt $^{3}$. Peyer und Brunner veröffentlichten dann nach eigenen, ausgedehnten Untersuchungen deren klassische Beschreibung.

\section{Biographische Notiz über J.C.PEYER ${ }^{4}$}

Johann Conrad Peyer wurde am 26. Dezember 1653 in Schaffhausen geboren. Er besuchte in seiner Vaterstadt das Collegium humanitatis. 1673 begann er an der Universität Basel das Studium der Medizin. Nach dem Tode seines Lehrers GLASER kehrte er 1675 nach Schaffhausen zurück. Hier verblieb er zwei Jahre und trieb unter der Leitung WePFERS eifrig anatomische und physiologische Studien. 1677 veröffentlichte P. sein klassisches Werk über die Glandulae intestinales, worin er die bis auf den heutigen Tag nach ihm benannten "plaques» beschrieb. Kurz darauf folgte ein längerer Studienaufenthalt in Paris, wo P. sich der Gönnerschaft des bekannten Anatomen DU VERNEY erfreute. 1681 promovierte er in Basel zum Doktor der Medizin, und im gleichen Jahre erfolgte auch seine Aufnahme in die «Academia Caesareo-Leopoldina», der naturwissenschaftlichen Akademie. In deren Publikationsorgan, den «Miscellanea curiosa sive Ephemerides», veröffentlichte P. zahlreiche Arbeiten. 1683 folgte ein zweiter, kurzer Aufenthalt in Paris. Hierauf widmete sich P. in Schaffhausen der Vollendung seines Werkes über die Wiederkäuer. Nach

${ }^{1}$ H.Fischer, Johann Jakob Wepfer, ein Beitrag zur Medizingeschichte des 17. Jahrhunderts, Zürich 1931.

${ }^{2}$ H. Nigst, Das anatomische Werk J.J. Wepfers, Sauerländer, Aarau 1947, S. 53.

${ }^{3}$ H.NIGST, l.c. S. 81.

4 B.Peyer, Der Arzt Joh. Con. Peyer, Zürich 1932.

C.Brunner und W.von Muralt, Aus den Briefen hervorragender Schweizer Ärzte des 17. Jahrhunderts, Basel 1919. 
1690 erlitt seine wissenschaftliche Lauf bahn auf medizinischem Gebiet einen jähen Abbruch, der endgültig bleiben sollte. Er wurde als Professor Eloquentiae des städtischen Gymnasiums seiner Vaterstadt gewählt. Dort wirkte er sechzehn Jahre. P. starb 1712.

\section{Die «Merycologia» und ihre Entstehungsgeschichte}

Im Jahre 1685 erschien in Basel das umfangreichste Werk P.s, die Merycologia sive de Ruminantibus et Ruminatione commentarius. Auch diese Arbeit ist der Erforschung der Verdauungsorgane gewidmet. Sie befaßt sich mit dem komplizierten Wiederkäuermagen. Das 288 Seiten umfassende, mit 12 Abbildungen ausgestattete Werk ist in drei Bücher gegliedert und mit einem Appendix versehen, in welchen P. Beiträge befreundeter Forscher aufnahm. Die Merycologia ist dem französischen Gesandten in der Schweiz, Tambonaeus, gewidmet.

Die Entstehung der Merycologia läßt sich bis auf die Kinderjahre P.s zurückverfolgen. In den Jahren 1664/65 hielt er sich im Valle Maggia auf, wo sein Vater damals das Amt eines ennetbirgischen Landvogtes bekleidete. Dort hielten die beiden eine Gemse, die nach den Worten P. (Merycol. S. 39) so zahm war, daß sie ihm wie ein Hund auf Schritt und Tritt folgte, wobei P. das. Wiederkauen beobachten konnte. Aus verschiedenen Stellen der Merycologia, vor allem aber aus dem regen Briefwechsel, den P. mit seinem Freunde von Muralt pflegte, geht hervor, daß es Wepfer war, der P. wiederholt auf die besonderen Verhältnisse des Verdauungstraktes der Wiederkäuer aufmerksam machte und ihn zur Erforschung dieses interessanten Gebietes ermunterte.

Im Oktober des Jahres 1675, nach der Unterbrechung seiner Basler Studienzeit, schreibt P. aus Schaffhausen an seinen Freund, den Zürcher Arzt vom Muralt ${ }^{5}$ :

«Neulich verschaffte uns unser trefflicher WEPFER alle Eingeweide einer Ziege zum Sezieren, damit wir sowohl dem Vorgang des Wiederkäuens als auch dem Werdeprozeß des Chylus genau nachforschten.»

Auf seiner im Jahre 1677 nach Paris unternommenen Reise machte $P$. in Genf einen Zwischenhalt. Hier erreichte ihn ein Brief WEPFERs ${ }^{6}$, in dem dieser von gelegentlich vorgenommenen Beobachtungen an Wiederkäuern berichtet.

${ }^{5}$ C. Brunner und W. von Muralt, l.c. S.158.

6 Merycologia, Append. Symbola II, S. 271-3. 
Bei Ziegen, Schafen und Rindern, die kurze Zeit nach der Fütterung geschlachtet wurden, fand Wepfer im Pansen Stengel und Gräser unverändert vor, während der Netzmagen schon feiner verarbeitetes Futter enthielt.

Dann teilt WEPFER am Schlusse dieses Briefes noch mit, daß ihm bei der Autopsie einer Kindsmörderin die innerste Schicht des Magens als drüsiges Gebilde vorgekommen sei, «glandularum congeries apparuit», eine Auffassung, die P. bei der Beschreibung des Wiederkäuermagens nutzbringend verwertete.

Im September 1678 berichtet P. aus Paris an von Muralt ${ }^{7}$ : «Was ich in meinen Mußestunden an der Ruminatio herumarbeite, das besteht hauptsächlich aus anatomischen Experimenten.»

Im Oktober des gleichen Jahres schreibt ihm WEPFER einen zweiten Brief $^{8}$.

Mit Genugtuung stellt WePFer fest, daß sich P. hin und wieder mit den Ruminantia beschäftige. Er ermahnt ihn, die Gelegenheit, im königlichen Tierpark Beobachtungen anzustellen, reichlich zu benutzen. Zu wenig sei über die Wiederkäuer bekannt. GESSNER und Aldrovand hätten sich mit dieser Frage nicht näher befaßt. Die ganze Verdauungsphysiologie könnte durch Untersuchungen in dieser Richtung bereichert werden. Es seien vor allem folgende Probleme zu studieren: die Nahrungspassage, der Mechanismus des Wiederkauaktes und die Anordnung der Fibrae, worunter er die Muskelfaserbündel versteht. Dann kommt er auf eine direkte Beobachtung der Wiederkäuerverdauung zu sprechen. Bei einer Kuh hatte sich infolge einer Hornverletzung an der rechten Flanke eine Fistel gebildet, die nach der Vermutung WePFERs mit dem Labmagen kommunizierte. Die Untersuchung der aus der Fistelöffnung hin und wieder kräftig hervorspritzenden Magenflüssigkeit, die er als einen «humor pultaceus» bezeichnet, brachte keine weiteren Resultate.

Das Studium der Wiederkäuerverdauung an einem Tiere mit einer Magenfistel beleuchtet eindrücklich den sicheren Blick WEPFERs für das Methodische. Erst im 19. und 20. Jahrhundert wurde zur experimentellen Untersuchung der Verdauungsvorgänge im Wiederkäuermagen bei Rindern eine Magenfistel angelegt (Flourens 1844, Wester 1926) ${ }^{9}$.

Auch in den «Ephemeriden» berichtet PEYer über das Fortschreiten seiner Arbeit. 1682 verkündete er $^{10}$ : «Etenim commentarium parare incepimus de ruminantibus.» Er fordert seinen Freundeskreis im Schoße der Akademie auf, ihm bei diesem Werke behilflich zu sein. Sein Appell war

${ }^{7}$ Brunner und von Muralt, l.c. S. 177.

${ }^{8}$ Merycol. Append. Symbola II, S. 273-6.

9 A.Czepa znd R.Stigler, Der Verdauungstrakt des Wiederkäuers im Röntgenbilde, aus Fortschr. naturwiss. Forsch. 1929, S. 1 und 2.

${ }^{10}$ Ephemerid. Med.-Phys. Germ. Acad. Nat. curios., II, Ann. I, 1682, obsv. 87. 
nicht vergeblich. P. stellte die Antworten seiner Freunde im «Appendix» der Merycologia zusammen.

Der Zürcher Arzt Johann Jakов WAgner beschreibt ${ }^{11}$ das Schleimhautrelief der Wiederkäuervormägen, die «carunculae» und «asperitates», und kommt dann auf die «fibrae» zu sprechen. Er weist auf das große Caecum der grasfressenden Tiere mit einfachem Magen hin.

Auch der Basler Arzt und Professor Johann Jaков Harder ${ }^{12}$ läßt seinen Kollegen von der Akademie nicht im Stich. Er unterstreicht die Wichtigkeit des Speichels für die Verdauung im Sinne einer fermentativen Einwirkung ${ }^{13}$. Der Inhalt des Blättermagens ist nach seiner Beobachtung viel feiner verarbeitet als das im Netzmagen vorgefundene Futter, eine durchaus richtige Feststellung.

Obgleich von Muralt durch eine «ampla praxis» sehr beansprucht ist, leistet auch er seinen Beitrag ${ }^{14}$. Er würdigt die Bedeutung der PEYerschen Untersuchungen für die Ernährungswissenschaft und kommt dann auf die reinen Tiere des alten Testamentes zu sprechen, eine Frage, die auch $P$. sehr am Herzen lag. Schrieb doch Peyer 1684 an von Muralt ${ }^{15}$ :

«Nach dem Zeugnis des Plinıus sezierten auch die Könige in Ägypten Leichname, um Krankheiten zu erforschen. Es ist also die Möglichkeit vorhanden, daß MosEs vermöge seiner anatomischen Kenntnisse die structura und dispositio ventriculorum in ruminantibus kannte und genau erforscht hatte, und deshalb wußte er auch als Naturkundiger, daß das Blut dieser Tiere reiner sei.»

Während seines zweiten Aufenthaltes in Paris schreibt er 1683 an voN MURALT $^{16}$ : «In meiner Mußezeit denke und arbeite ich jetzt schon wieder an der Ruminatio herum, ein Stoff, der nützlich und nicht undankbar ist, wenn er auch für trivial gilt.» $\mathrm{P}$. befürchtete offenbar, daß seine Zeitgenossen dieser Arbeit nicht das nötige Interesse entgegenbringen könnten.

Zu Anfang des Jahres 1684 ist P. endlich so weit. Erleichtert teilt er voN Muralt mit ${ }^{17}$ : «Meine Merycologia ist schon längst zu Ende geführt, bis jetzt aber fehlen noch die Illustrationen, deren Verfertigung der Basler Kupferstecher TURNEYSER länger als billig hinausschiebt.»

11 Merycol. Append. Symbola III, S. 276/7.

${ }^{12}$ Merycol. Append. Symbola IV, S. 278-83.

${ }^{13}$ Der Speichel der Wiederkäuer enthält keine diastatischen Fermente.

14 Merycol. Append. Symbola V, S. 283-5.

15 Brunner und von Muralt, l.c. S. 218.

${ }^{16}$ Brunner und von Muralt, l.c. S. 215.

${ }^{17}$ Brunner und von Muralt, l.c. S. 221. 
Im Alter von 32 Jahren konnte dann P. seine umfangreichste Arbeit der Öffentlichkeit übergeben.

\section{Peyers «Merycologia» in ihrem Verhältnis zur früheren Literatur über die Wiederkäuer}

P. unternimmt in der Merycologia den Versuch, die Biologie der Wiederkäuer und des Wiederkauens möglichst umfassend darzustellen. Zunächst untersucht er das Vorkommen des Wiederkauens innerhalb der verschiedenen Tierklassen. Dann bemüht er sich, die Wiederkäuer im engeren Sinne zu klassifizieren. Hierauf beschreibt er die Topographie, Anatomie und gewebliche Struktur des Wiederkäuerapparates und versucht in diesem Zusammenhange den Anteil der verschiedenen Mägen am Verdauungsvorgang zu bestimmen. Auch dem Mechanismus des Wiederkauens widmet er längere Ausführungen. Darüber hinaus bemüht er sich, die Bedeutung der Wiederkäuer innerhalb des von Gott in seiner Allmacht und Weisheit geschaffenen Tierreiches zu erkennen. Diese «automata divina» sind ganz und gar nicht minderwertige Wesen. Der Schöpfer hat sie nach der Überzeugung P's. mit wunderbar ausgedachten Verdauungswerkzeugen versehen.

Mit voller Berechtigung stellt $P$. fest, daß er erstmals eine gesamthafte Darstellung der Ruminantia gegeben habe.

Die erste wissenschaftliche Bearbeitung geht auf ARIstoteles zurück ${ }^{18}$. Dieser zählte alle horn- und geweihtragenden Paarhufer mit fehlenden oberen Vorderzähnen zu den Wiederkäuern. Außerdem besäßen dieselben einen vierkammerigen Magen. Über diese auch heute noch gültige Einteilung hinaus gab Aristoteles eine prachtvolle Beschreibung der einzelnen Mägen, wobei er sich für die Nomenklatur derselben auf die Beschaffenheit der Schleimhautoberfläche stützte. So nennt er den zweiten Magen Netzmagen, den dritten Igelmagen. Die Vierzahl der Mägen erklärte sich ARIstoteles aus dem Fehlen der oberen Vorderzähne, welches eine besonders gründliche kompensatorische Magenverdauung erheische.

Auch GALEN beschäftigte sich gelegentlich mit den Wiederkäuern. Er studierte an einem Zicklein ${ }^{19}$, das er in strengster Isolierung von seinen Artgenossen aufzog, den Übergang von der Ernährung mit flüssigen Spei-

${ }^{18}$ Aristoteles, Hist. Anim. II, 17; De Part. Anim. III, 14, III, 2; J.A.Smith und W.D.Ross, The Works of Aristotle, Vol. IV, V, Oxford 1910, 1912.

${ }^{19}$ GaLeN, De loc. aff. VI, Kp. 6 (KüHN VIII, S. 442-4). 
sen zur Fütterung mit fester Kost. Dabei stelle sich auf wunderbare Weise das Wiederkauen von selbst ein.

Die Zoographen des 16. Jahrhunderts ${ }^{20}$ greifen dann das Problem des Wiederkauens wieder auf und führen endlose Diskussionen über die Bedeutung, die dem Fehlen der oberen Schneidezähne zukomme.

Volcher CoITer (1573) untersuchte im Rahmen einer vergleichend anatomischen Studie des Quadrupedenskelettes auch das Knochengerüst der Wiederkäuer ${ }^{21}$.

Ulisses Aldrovandus, einer der bedeutendsten Zoographen des 16. Jahrhunderts, unterscheidet schon die mechanische Funktion der Vormägen von der eigentlichen Verdauungsarbeit (concoctio) des Labmagens ${ }^{22}$. Er veröffentlicht eine der ersten Abbildungen des Wiederkäuermagens, wobei er zum Zwecke der Darstellung das Schleimhautrelief an die Magenoberfläche projiziert.

Fabricius ab Aquapendente (1618) beschäftigte sich mit dem Mechanismus der Rejektion, den er auf das Wirken der Galenischen «facultates naturales» zurückführte ${ }^{23}$. Er erkannte, daß die Milch beim säugenden Wiederkäuer direkt in den Labmagen fließt.

Francis Glisson (1677) ließ die Galenische Theorie der Naturkräfte fallen und führte das Wiederaufsteigen der Nahrung auf eine retroperistaltische Bewegung des Magens zurück ${ }^{24}$. Bemerkenswert ist seine vergleichend anatomische Deutung des Labmagens, den er dem menschlichen Magen gleichsetzt. Diese Erkenntnis gebührt übrigens SEverino, der schon 1645 den Labmagen als den «ventriculus proprie dictus» bezeichnet hatte.

Die vor dem Erscheinen der Merycologia bedeutendste Arbeit über den Wiederkäuermagen ist die 1681 publizierte, vergleichend anatomische Studie des Magendarmkanals von Nehemiah GREW ${ }^{25}$. Er beschrieb «pointed knots» auf der Schleimhaut der Vormägen, deutete die Pansenpfeiler als vorstehende Muskelwülste und erkannte die Homologie des Labmagens mit dem menschlichen Magen. Der vierte Magen allein, so erklärte er, besitze Drüsen. Damit gab er zum ersten Male eine klare, auf morphologische Merkmale gestützte Begründung dieser Homologie.

\footnotetext{
${ }^{20}$ R. BuRckhaRd, Geschichte der Zoologie, Leipzig 1907, S. 52.

${ }^{21}$ V. CoIter, De Quadrupedum sceletis, Nürnberg 1573.

${ }^{22}$ U.Aldrovandus, Quadrupedum omnium bisulcorum Hist., Frankfurt 1647, S. 1-2, 165.

${ }^{23}$ Fabricius ab Aquapendente, De variet. ventr., S. 131-8, aus Op. omn. anat. et physiol.,

${ }^{24}$ F. Glisson, Tract. de ventr. et int., Amstld. 1677, S. 140-5.

[Leipzig 1687.

${ }^{25}$ N. Grew, The compar. Anat. of Stom. a. guts, London 1681, S. 16-30.
} 


\section{Der Inhalt der «Merycologia»}

\section{A. ERSTES B U GH}

Im ersten Buche, das «De ruminantium speciebus et differentiis» betitelt ist, versucht P. nachzuweisen, daß neben den eigentlichen Wiederkäuern auch in anderen Tierklassen gewisse Vertreter in der Ausbildung ihres Magendarmkanals Analogien mit den Ruminantia aufweisen.

Bei der Untersuchung der Insekten stützte er sich auf zeitgenössische zootomische Publikationen, in denen schon die Frage erörtert wurde, ob bei gewissen Insekten mit vielfachem Magen ein Wiederkauen anzunehmen sei. Von MURALT ${ }^{26}$ hatte 1682 die inneren Organe der Maulwurfsgrille beschrieben. In einer äußerst sorgfältigen Studie desselben Insektes überprüfte P. die Angaben von Muralts (S. 8) und machte ihn darauf aufmerksam, daß er es unterlassen habe, die beiden letzten «ventriculi» zu erwähnen, womit $P$. die beiden entodermalen Blindsäcke meint, die er sogar als dem Pankreas analoge Organe ansieht. Dann schlug er für die erste Ausstülpung des Verdauungskanales den Namen «ingluvies» vor, eine auch heute gebräuchliche Bezeichnung. Das morphologische Talent P.s bewährte sich auch bei seinen entomologischen Untersuchungen. Ein eigentliches Wiederkäuen, so führte er weiter aus, könne zwar bei den Insekten nicht angenommen werden, denn die Speisen werden ja nicht ein zweitesmal gekaut. Aber die sukzessive Verarbeitung der Nahrung in dem komplexen Magenapparat der Gryllotalpa weise doch Parallelerscheinungen mit der Wiederkäuerdigestion auf.

Von Muralt beherzigte die Kritik seines Freundes. In seiner neuen, mit prächtigen Abbildungen versehenen, in den «Ephemeriden» des Jahres 1683 herausgekommenen Beschreibung der Gryllotalpa unterscheidet er am Magendarmkanal die «ingluvies», den Magen und zwei «appendices», die er auch «ventriculus tertius et quartus» nennt ${ }^{27}$.

Eingehend bespricht dann P. die umfangreiche Literatur, die seit der Aristotelischen Beschreibung des wiederkäuenden «Skaros» über ruminierende Fische entstanden war. Dabei kommt er zu keinem abschließenden Urteil.

Auch bei den Vögeln untersuchte P. die Verdauungsorgane. Schon 1683 hatte er in den «Ephemeriden» eine Beschreibung des Storchenmagens publiziert ${ }^{28}$. Er beschreibt denselben als ein doppeltes Gebilde, das sich aus einem «sinus glandulosus» und dem eigentlichen Muskelmagen zusammensetze. Die kräftige Muskulatur, eine «valvula rugosa» am Pylorus und der weite Magenmund schienen P. auf die Möglichkeit eines Wiederaufsteigens der Speisen hinzuweisen.

Am Ende des ersten Buches kommt dann P. auf die eigentlichen Wiederkäuer die «vere ruminantia», zu sprechen. Er zieht dabei Aristoteles, Plinius, Caesar und noch viele andere Autoren zu Rate und setzt sich auch mit den neueren zoographischen Werken auseinander. P. bemüht sich, eine kritische Sichtung des großen literarischen Materials vorzu-

${ }^{26}$ Ephemerid. Dec. II, Ann. I, 1682, obsv. 62.

${ }^{27}$ Ephemerid. Dec. II, Ann. II, 1683, obsv. 30.

${ }^{28}$ Ephemerid. Dec. II, Ann. II, 1683, obsv. 97. 
nehmen. Er unterscheidet den Auer- oder Urochsen vom Wisent ${ }^{29}$ und bespricht sogar afrikanische Boviden. Er ist bestrebt, die Klassifizierung auf exakte morphologische Merkmale zu stützen. Wie kritisch er dabei vorgeht, beweist die Besprechung des Renntieres. Da er nicht selber Gelegenheit hatte, ein solches zu untersuchen, stützte er sich auf den Bericht eines Johann Scheffer, den «novissimus Lapponiae scriptor» (S. 34/5). Dieser habe die Renntiere als geweihtragende, nichtwiederkäuende Paarhufer geschildert. Dies erscheint nun P. sehr zweifelhaft. Denn das Vorkommen eines Geweihes bei einem Paarhufer weise doch deutlich die Merkmale eines Wiederkäuers auf. Allerdings gehöre zur Sicherstellung dieser Diagnose noch eine anatomische Untersuchung des Magens. Eine zuverlässige Schilderung der Verdauungsorgane dieser Tiere sei aber bis jetzt nirgends aufzutreiben.

Die Einteilung der «species caprarum» bereitete P. die größten Schwierigkeiten, «major est difficultas quam superare conabor».

\section{Kurze Skizzierung der heutigen Kenntnisse über den Wiederkäuermagen}

Vor der Besprechung des zweiten Buches der Merycologia sei eine kurze Skizzierung der modernen anatomischen und physiologischen Kenntnisse über den Wiederkäuermagen vorangestellt ${ }^{30}$.

Der Wiederkäuermagen setzt sich aus drei Vormägen und dem Labmagen zusammen. Die beiden ersten Vormägen, der Pansen und die Haube, bilden sich aus dem kardialen Magenblindsack, der Psalter oder Blättermagen aus einer weiter pyloruswärts gelegenen Corpuspartie, während der Labmagen dem einfachen Drüsenmagen des Menschen und der einmagigen Tiere homolog ist. Die Vormägen besitzen im Gegensatz zum Labmagen keine Drüsen. Ihre Schleimhaut wird von einem verhornten Pflasterepithel ausgekleidet.

Der größte Vormagen, der Pansen, wird an seiner Außenfläche durch die Pansenfurchen unterteilt, die innen den vorspringenden, aus Muskelwülsten gebildeten Pansenpfeilern entsprechen. Der Pansen besitzt ein pigmentiertes Plattenepithel. An der Schleimhaut springen makroskopisch sichtbare Papillen hervor, die auf den Pansenpfeilern fehlen.

Die Schleimhautoberfläche des Netzmagens ist in polygonale, von Leisten umgrenzte Felder eingeteilt, auf denen sich ebenfalls Warzen erheben.

Der Psalter oder Blättermagen ist von kugeliger Form. Sein Lumen wird von Schleimhautfalten durchsetzt, die von oben und von der Seite wie die Blätter eines Buches in das Innere des Psalters hineinragen und nur den schmalen Boden freilassen.

${ }^{29}$ Schon Conrad Gessner hatte auf die Verwechslung des Auerochsen mit dem Wisent hingewiesen. Er hat in seinem Tierbuch beide abgebildet, C. Gesnerus, De quadrupedibus viviparis, Ed. sec., Frankfurt 1603, S. 128, 145.

${ }^{30}$ Ellenberger-Baum, Handbuch der vergleichenden Anatomie der Haustiere, 18. Auflage, Berlin 1943. - Handbuch der mikroskopischen Anatomie des Menschen, hg. v. W. von MöLLENDOrF, 5. Bd. 1927-1936. - W.Nusshag, Lehrbuch der Anatomie und Physiologie der Haustiere, Leipzig 1949. 
Die Muskulatur der Vormägen setzt sich aus zwei Schichten glatter Muskeln zusammen, die besonders im Pansen und der Haube kompliziert verlaufen. Die Psalterblätter werden von der dicken inneren, zirkulär verlaufenden Muskelschicht mit Muskelzügen versorgt.

Die Vormägen mit ihrer rauhen Schleimhautoberfläche bewirken eine mechanische Zerkleinerung der Nahrung. Das Futter wird hier auch mazeriert und durch Einwirkung von Infusorien aufgelockert, worauf dann eine bakterielle Zersetzung der Celluslose in Methan und Kohlensäure erfolgt.

Der Labmagen ist der drüsige, fermentbildende Magenabschnitt. Die feste Nahrung gelangt zuerst in den Pansen. Von dort wird sie zum Wiederkäuen in den Mund hinaufbefördert. Die wiedergekaute Nahrung gelangt wiederum in den Pansen und in die Haube zurück. Sobald die Speisen im Pansen und Haube genügend zerkleinert sind, vermögen sie die enge, schlitzförmige Öffnung zwischen Haube und Psalter zu passieren. Hier erfolgt eine Auspressung der Nahrung, worauf dann der Chymus in den Labmagen weiterbefördert wird.

Die Milch fließt beim säugenden Wiederkäuer durch die Speiserinne direkt in den Labmagen. Die Schlund- oder Speiserinne ist die physiologische Fortsetzung der Speiseröhre und verläuft durch die Haube bis zur Psalteröffnung. Dabei schließen sich ihre Lippen reflektorisch zu einer Röhre, noch ehe das Getränk sie berührt hat. Die Speiserinne wird von kräftigen Muskelzügen umfaßt.

\section{B. ZWEITES BUGH}

Den Höhepunkt der Merycologia bildet deren zweiter Teil, den P. «De Partibus Ruminationi inservientibus» betitelt hat.

Befreit vom Ballaste der alten Literatur, rückt er der Materie mit eigener, exakter Forschung zu Leibe und kann seine Untersuchungsergebnisse mit früheren und zeitgenössischen Angaben vergleichen.

Zunächst gibt er einen allgemeinen Überblick über den Ruminationsapparat. Dieser setzt sich aus Haupt- und Hilfswerkzeugen zusammen. Die für das Wiederkauen unerläßlichen Organe sind die Mägen, die Speiseröhre und die Mundwerkzeuge. Sie werden in ihrer Funktion unterstützt durch das Zwerchfell und die Bauchmuskeln.

Die Topographie der Wiederkäuermägen ist kompliziert. Sie füllen einen großen Teil des Abdomens aus und können bis ins Becken hinunterziehen. Trotz ihrer engen Beziehungen zu den Nachbarorganen, dem Zwerchfell, der Leber, der Milz und dem Netz, sind sie frei beweglich.

Die Magenwand setzt sich aus drei membranösen und einer fibrösen Schicht zusammen. Die innerste Schicht hält P. für eine «membrana glandulosa» oder eine «glandula instar membranae expansa» (S. 79). Entschieden weist er die Ansicht Brunners zurück, der die «membranae secretoriae» nicht zu den eigentlichen Drüsen gezählt habe. Die zweite Schicht, die «tunica fibrosa», ist nichts anderes als ein kreisförmig angeordneter Muskel. Der spiralige Verlauf dieser «fibrae musculosae» sei vor allem nach dem Auskochen gut sichtbar. Hier bedient sich P. einer damals allgemein üblichen Technik, die auch für die Untersuchung der parenchymatösen Viscera verwendet wurde. 
Vier Gefäßarten (S. 85) versorgen die Magenwand. Die Arterien führen ernährendes Blut und Wärme heran. Außerdem liefert das arterielle Blut noch das Material zur Magensaftbildung.

Die Lymphgefäße leiten den Überfluß an sezernierter Magenflüssigkeit in die Cisterna ab, um den Chylus zu verdünnen, der nach der Meinung P.s bei den Wiederkäuern eine besonders dicke Konsistenz aufweise (S. 90). Die der äußeren Magenwand anliegenden Lymphknoten, die P. nach der von Sylvius 1663 eingeführten Nomenklatur als «Glandulae conglobatae» bezeichnet, dienen der Lymphflüssigkeit nur als Durchgangsstation, «tantummodo transitum praebent».

Die Innervierung der Mägen erfolgt durch das achte Nervenpaar von WiLlis (N. vagus).

Die Magenvenen führen das Blut durch die Leber zum Herzen und besitzen keine resorbierende Funktion.

\section{Allgemeine Verdauungstheorie}

Die allgemeine Verdauungstheorie P.s ist eine durchaus eklektische. Die Aristotelische und Galenische Wärmetheorie, die von van Helmont und Sylvius DE LE Bö̈ formulierte Fermentationstheorie vereint er mit den zu seiner Zeit immer mehr an Boden gewinnenden iatromechanischen Vorstellungen, nach denen der Verdauungsprozeß auf physikalische Vorgänge reduziert wurde. Er kommt dabei trotz der zu seiner Zeit fehlenden Kenntnisse in organischer Chemie überraschend nahe an die heutigen Auffassungen über die Verdauung der Wiederkäuer heran. So verbindet er den Akt des Wiederkäuens mit den beiden ersten Mägen, wo eine Mazeration und mechanische Zerkleinerung der Speisen erfolge, und verlegt die eigentliche chemische Einwirkung in den Labmagen, wo eine «temperatio und collectio» des Chylus einsetze (S. 99).

In diesem Zusammenhange weist er auch auf die besonders große Muskelkraft der Wiederkäuermägen hin. So habe er seinerzeit in Paris im Beisein Du Verneys im Herzen einer Kuh eine Nadel gefunden. Auch die Magenwände dieses Tieres seien mit Nägeln bespickt gewesen (S.95/6). Dies alles sei nur durch eine kräftige Peristaltik zu erklären. P. streift hier also sogar veterinärmedizinische Fragen.

Nach dieser allgemeinen Einführung in Struktur und Funktion des Wiederkäuermagens folgt die Detailbeschreibung der einzelnen Mägen.

\section{Der Pansen}

P. erkennt die beträchtliche Ausdehnung dieses größten Magens. Der Pansen füllt den größten Teil der Bauchhöhle aus, «praecipuam abdominis molem adimplens» (S. 107).

Die äußere Fläche ist glatt und wird durch einen «sulcus» in zwei Hemisphären geteilt. Neben der Einmündung der Speiseröhre ist eine kleine Erhebung zu beobachten, «monticulus prope gulam», womit $\mathrm{P}$. wohl den gemeinsamen Magenvorhof bezeichnet. 
Nach Injektion von gefärbtem, mit Öl und Terpentin vermischtem Wachs studiert er den Gefäßverlauf in der Magenwand (S. 110). Besonders dicht sind die Gefäßmaschen in der inneren Wandschicht.

Zur Darstellung der Muskelschichten mazerierte P. die Mägen in warmem Wasser. Hierauf sorgte er durch Einblasen von Luft oder durch Auffüllen mit Wasser (S. 111) für die nötige Turgeszenz und präparierte dann die einzelnen Muskelschichten. Er vergleicht die «tunica musculosa» mit dem Herzmuskel. Die Kontraktion dieser Muskeln treibt die Speisen nach oben. Er unterscheidet die sich überkreuzenden (S. 115/6) äußeren und inneren Muskelschichten. Sorgfältig hat er dieselben auf den dem zweiten Teil seines Werkes beigefügten Abbildungen dargestellt (Abb. II und III).

Die dritte Schicht, die «tunica membranacea» (S. 116) oder «nervea», setzt sich aus den ausgebreiteten Nerven und Blutgefäßen sowie aus den sehnigen Enden der «fibrae» zusammen. Diese Schicht besitzt eine besonders reichliche Gefäßversorgung. Kleine Zungen der «tunica nervosa» bilden papillenartige Körperchen. Diese erheben sich wie Schwerter und sind von einer Scheide umhüllt. Die Funktion dieser Papillen sieht P. in der mechanischen Zerkleinerung der Nahrung mit Hilfe der kräftigen Magenperistaltik.

Die vierte Schicht, die «tunica crustosa», läßt sich nach ihrer Mazerierung leicht ablösen. Sie ist mit «apices» versehen, worunter P. die heute als Warzen oder Zotten bezeichneten Gebilde versteht. Die «apices» sind an ihren Enden bald gefranst, bald stumpf. Ihre Farbe spielt vom Weißen ins Schwarze (S. 118). Ausdrücklich stellt P. fest, daß die «crusta» ähnlich wie die menschliche «cuticula» oder «epidermis» keine Gefäße besitze (S. 119: «Nulla vasa conspicua per tunicam hanc crustosam feruntur, ut neque per cuticulam seu epidermida qua cutis humana obtegitur»). Trefflich hat somit P. das pigmentierte Pflasterepithel des Pansen beschrieben.

Die Frage, ob diese «cuticula» ein Ferment ausschwitze, läßt P. offen. Genau ist auch die zeichnerische Darstellung der Pansenpfeiler mit ihren fehlenden «apices» (S. 187, Abb. IV). Die Pfeiler selbst bezeichnet er als «chordae» oder «fornices».

\section{Die Haube}

In der Wand des zweiten Magens, dem Netzmagen oder Haube, konnte P. eine ähnlich starke Muskelschicht wie beim Pansen nachweisen. Er beschreibt ferner einen «canaliculus singularis» (S. 124), der von zwei Wülsten («valvulae geminae») begrenzt werde und von der Einmündung des Oesophagus zur unteren Netzmagenöffnung verlaufe. Zu diesem Kanal ziehen kräftige Muskelbündel hin, die P. auch auf der Abb. V dargestellt hat (S. 187). Damit hat er aber erstmals eine zuverlässige Beschreibung der Speiserinne gegeben, deren Lippen und Muskelzüge er schildert. Dann beschreibt er die Schleimhautoberfläche mit ihren polygonalen Feldern, die er «areolae» nennt (heute Cellulae reticuli genannt). Diese sind von «sepes» umgrenzt (den heute als Haubenleisten bezeichneten Erhebungen). In den areolae schildert P. die eingepflanzten «apices». Die Öffnung der Haube gegen den Pansen hin ist größer als ihre Kommunikation mit dem Blättermagen (S. 132).

\section{Der Psalter}

Auch dem dritten Magen, dem Psalter, widmet P. eine eingehende Beschreibung. Er schildert dessen verschiedene Größenverhältnisse bei den einzelnen Haustieren und beobachtet richtig, daß die Mukosa zwischen Haube und Psalter eine rauhe Oberfläche auf- 
weist (S. 137), während sie am Übergang vom Blättermagen zum Labmagen glatt ist. P. unterstreicht die reichliche Blutversorgung der Blätter, erkennt deren verschiedene Größenordnung und schildert ihre Wärzchen. Er unterscheidet genau die schwächere äußere Längsfaserschicht von der stärkeren inneren Muskelschicht. Die innere Schicht versorgt auch die Blätter mit Muskelzügen. An den Blättern selbst ist es P. gelungen, die in der Blattrichtung verlaufenden Muskelbündel von den aus dem Blattansatz zum freien Blattrand hinziehenden Muskelfasern zu unterscheiden (S. 139: «aliae [fibrae] enim secundum foliorum longitudinem protenduntur, quas in fasciculos collectas superum edit firmatque orificium, aliae contrario situ per foliorum altitudinem assurgunt, priores itidem decussaturae»).

\section{Der Labmagen}

Den vierten oder Labmagen vergleicht $P$. ebenfalls mit dem einfachen Magen des Schweines, Hundes oder Menschen. Die Homologie derselben hat er allerdings nicht so scharf herausgearbeitet wie GREw, der das Vorkommen der Drüsen als Kriterium benutzt hatte.

P. beobachtet die reichliche Gefäßversorgung (S. 145) und die besondere Dicke (S. 99, 146) und Weichheit der mit Schleim überzogenen «crusta» des Labmagens. In diesem Falle versteht er unter der «crusta» das der Schleimhaut entsprechende Stück der Magenwand. Die Milch, so sagt P., gerinnt hier, um sich nachher wiederum aufzulösen (S. 146: «lac in abomaso ocyus firmiterque coagulat, et post iterum resolvitur»). Er führt diesen Vorgang auf ein neu hinzutretendes Ferment zurück. Denn durch Wärme allein sei diese Auflösung nicht zu erklären. Sie werde bewirkt durch ein «fermentum novum, cuius vis ab acore volatili maxime dependeat» (S. 150).

Diese Formulierung erinnert sehr an den «acor specificus» vaN HeLMonTs, den derselbe aber, noch ganz im Banne Galenischer Anatomie, als ein Milzferment bezeichnete, das über eine Vene von der Milz zum Magen fließe. P. erkennt die sekretorische Funktion der Magenschleimhaut und die auflösende Wirkung des Magensaftes auf die Milch. Diese Auffassung des Magensaftes als eines spezifischen Sekretes wurde damals aber im allgemeinen nicht erkannt. Auch die saure Reaktion wurde erst zu Anfang des 19. Jahrhunderts endgültig anerkannt ${ }^{31}$.

Bemerkenswert ist auch, daß P. die chemische Funktion des Labmagens gegenüber der vorwiegend mechanischen Aufgabe der Vormägen abgrenzt.

\section{Die Speiseröhre}

Die Speiseröhre ist ein «musculus cylindraceus». P. erkennt die sich überkreuzenden, spiralig verlaufenden Muskelfasern (S. 162). Die Speiseröhrenmuskulatur gewährleiste infolge ihrer kräftigeren Beschaffenheit und der spiraligen Anordnung ihrer Fasern einen schnelleren Durchtritt der Speisen als die Magendarmmuskulatur ${ }^{32}$.

31 Tiedemann und Gmelin, Versuche über die Wege, auf welchen Substanzen aus dem MagenDarmkanal ins Blut gelangen, Heidelberg 1820, S. 56.

32 Ellenberger und Baum, $l$. c. S.395: «sie (die Muskelschicht der Speiseröhre) besteht bei Hd. und Wdk. bis zum Magen aus roter, quergestreifter Muskulatur». 


\section{Der Kauapparat}

Das Fehlen der oberen Schneidezähne ist für die Wiederkäuer spezifisch, «certissimum ruminationis indicium». Die einwurzeligen Schneidezähne und die verästelten Wurzeln der Backzähne werden beschrieben. P. vermerkt, daß die Eckzähne sowohl oben als auch unten fehlen ${ }^{33}$.

\section{Der Hilfsapparat}

Das Zwerchfell umgreift mit kräftigen Muskelzügen die Speiseröhre. Dieselben wirken als Kontrollstation für den wiederzukäuenden, nach oben gleitenden Bissen (instar sphincteris). ${ }^{34}$ Den Rejektionsakt faßt $\mathrm{P}$. als eine retroperistaltische Bewegung auf, die durch die Bauchpresse unterstïtzt werde (S. 177). ${ }^{35}$

\section{Der Speiseweg}

Überraschend modern mutet uns die Beschreibung der Nahrungspassage an. Das zuerst geschluckte Futter gelangt in den Pansen, hierauf wird es in den Mund zurückbefördert. Die wiedergekäute Nahrung gleitet wiederum in den Pansen zurück (S. 120). ${ }^{36}$ Die feine Öffnung zwischen Haube und Psalter ist nur für die wiedergekaute, fein zerteilte Nahrung durchlässig. Die gleiche Beobachtung hatte auch schon HARDER mitgeteilt. Sie ist auch heute noch gültig.

Dem Psalter schreibt P. eine ausschließlich mechanische Funktion zu. Der Nahrungsbrei wird in demselben trockener, weil die flüssigen Bestandteile durch diesen Preßmagen in den Labmagen ausgespritzt werden. Ferner wird hier der Nahrungsbrei zerkleinert, wobei die Muskelblätter mit ihren Warzen wie eine Feile einwirken (S. 139: «Quamobrem echinum [ = Psalter $]$ appositae et limae comparabimus et pressorio seu torculari, quo ruminatum digestumque pabulum comprimitur»). ${ }^{37}$

Den Speiseweg der Milch beim Wiederkäuersäugling schildert P., im Gegensatz zu FABRICIUS, nicht richtig. Nach seiner Auffassung passiert die Milch alle Mägen, bevor sie sich in den Labmagen ergießt. Die anatomische Beschreibung der Speiserinne hat P. hingegen erstmals gegeben.

${ }^{33}$ Ellenberger und Baum, l.c. S.383: «Der äußerste (Eckschneidezahn) ist dem Caninus homolog.»

${ }^{34}$ Neuerdings wird angenommen, daß diese Muskelzüge des Zwerchfells zur Offenhaltung der Speiseröhre dienen. Vgl. W. Nusshag, l.c. S. 231/2.

${ }^{35}$ Diese Auffassung ist erst in neuerer Zeit aufgegeben worden, wobei für die Rejektion das physiologische Druckgefälle vom Magen zur Speiseröhre hin geltend gemacht wurde. Für das Aufsteigen der Speisen vom Magen in den Oesophagus genüge darum ein Offenhalten der Cardia ohne Magenperistaltik und Bauchpresse. Vgl. Nusshag, l.c. S. 231, Handwörterbuch der Naturwissenschaften, Bd. 6, S. 637.

${ }^{36}$ Diese Anschauung wurde erst in neuerer Zeit mit Hilfe von Kontrastaufnahmen bestätigt (Czepa und Stigler, 1929).

${ }^{37}$ Eine ähnliche Ansicht wird bestätigt durch W.Nusshag, l.c. S. 232 und im Handbuch der vergleichenden Anatomie der Wirbeltiere, III. Bd., Berlin 1937, S. 539. 


\section{G. DRITTES B UCH}

Im dritten und letzten Teil seines Werkes befaßt er sich mit der allgemeinen Biologie des Wiederkäuens, wobei er auf die nach seiner Meinung besonders feine Chylifikation im Wiederkäuerorganismus hinweist. Diese sei nur durch die sorgfältige Elaboration im komplizierten Wiederkäuerapparat möglich. Er faßt also denselben nicht nur als eine Adaptation an die besonders harte, schwer zu erschließende Heu- und Grasnahrung, sondern auch als einen zu optimaler Digestion befähigten Organkomplex auf. Darum aßen die Juden auch nur das Fleisch wiederkäuender Tiere.

Im dritten Buche ergeht sich P. weiterhin in langwierigen, naturphilosophischen und theologischen Spekulationen.

Dann streift er kurz die Pathologie des Wiederkäuens. Die Rumination werde durch Krankheit, aber auch durch ein Zuviel oder ein Zuwenig an Nahrung gestört.

Auch dem menschlichen Wiederkäuen, von dem P. schon im ersten Buche der Merycologia einige Beispiele angeführt hatte, widmet er einen Abschnitt. Er unterscheidet dreierlei Arten der «ruminatio humana»: eine angeborene, wobei der Gemütszustand der Mutter einen großen Einfluß ausübt, eine durch Angewöhnung erworbene und schließlich eine bei pathologischen Prozessen auftretende, dem Brechen ähnliche Rumination.

\section{Kritische Würdigung der «Merycologia»}

Die eigentliche Leistung P.s liegt in der noch nie erreichten Detailbeschreibung der Topographie, Anatomie und Histologie des Wiederkäuermagens. Wohl hatten schon frühere Forscher auf die «asperitates» und «pointed knots» der Schleimhaut hingewiesen. Aber die Verteilung, Form und Färbung der Zotten ist vor P. nie so genau beschrieben worden. Völlig neu ist auch die Beschreibung der polygonalen Haubenfelder und der sie umgrenzenden Leisten und die minutiöse Schilderung des Muskelverlaufes innerhalb der Psalterblätter.

Neuartig ist auch die zeichnerische Darstellung des Wiederkäuermagens. Aldrovandus und Grew waren nicht über eine schematische oder halbschematische Wiedergabe hinausgekommen. P. zeichnet hingegen in naturgetreuer Ausführung die äußere Magenform. Auch ist ihm eine sorgfältige zeichnerische Darstellung der inneren und äußeren Muskelschichten der Vormägen auf separaten Tafeln geglückt.

HaLler hatte schon von der Merycologia gesagt: «Caeterum ventriculos animalium ruminantium copiose et abunde describit, internas omnium asperitates, fibras musculosas, has primus.» ${ }^{38}$

Von einem Gebrauche des Mikroskopes ist nirgends die Rede. P. geht nicht über das makroskopisch oder mit Hilfe der Lupe Feststellbare hinaus.

${ }^{38}$ B. Peyer, l.c., S. 337, Anm. 63. 
Die Funktion des komplexen Wiederkäuermagens leitet P. einmal aus den Resultaten der anatomischen und geweblichen Untersuchungen ab. Sodann stützte er sich, ähnlich wie es WEPFER getan hatte, auf die Beschaffenheit des Nahrungsbreies in den einzelnen Mägen geschlachteter Tiere, die Rückschlüsse auf die Funktion der einzelnen Magenabschnitte zu ziehen erlaubte. Überraschend gut gelingt ihm dabei die Unterscheidung der mechanischen Funktion der Vormägen von der chemischen Einwirkung des Labmagens.

CoLE schreibt in seiner Geschichte der vergleichenden Anatomie ${ }^{39}$, daß das Werk P.s über den Wiederkäuermagen als eine der besten früheren Bearbeitungen dieses Gebietes zu betrachten sei. Es war also wohl der Mühe wert, dieses umfangreichste, auf sorgfältigen Untersuchungen aufgebaute und mit erschöpfender Angabe der früheren Literatur versehene Werk Peyers in Erinnerung zu rufen.

\section{Prognostikation auf 24 zukünftige Jahre von Theophrastus Paracelsus und ein zeitgenössischer Deutungsversuch}

Von Bernhard Milt, Zürich

Vor etlichen Jahren erhielt der Verfasser dieser Zeilen einen Brief aus Frankreich von einem Unbekannten, mit der Anfrage, ob es richtig sei, daß diese Prognostik Theophrasts gerade die Zeit nach dem letzten Kriegsende im Auge gehabt habe. Es muß damals in unserm Nachbarland eine Schrift erschienen sein, welche eben dieses behauptete. Das Schriftchen konnte durch unsern Buchhandel nicht aufgetrieben werden, und so hat der Schreiber seinen Titel und Verfasser längst vergessen. Ihn interessierte in der Folge mehr die kleine Arbeit von Paragelsus, die nach mehr als vier Jahrhunderten aktuell sein sollte.

Sudhoff hat sie in den 10. Band seiner Paracelsus-Ausgabe aufgenommen; er bezeichnete sie einmal als politisch-mantische, ein andermal als religionspolitisch-philosophische Arbeit, in der Einleitung (S. XLII) auch als politische Voraussage. Über Inhalt und Sinn des Werkleins war er sich offenbar nicht völlig klar. Der genauere Titel lautet:

39 J.F. CoLe, A History of comparative Anatomy, London 1944, S. 251. 